\title{
Recurrent secondary postpartum hemorrhages due to placental site vessel subinvolution and local uterine tissue coagulopathy
}

Pavol Zubor ${ }^{1,3^{*}}$, Karol Kajo $^{2}$, Karol Dokus ${ }^{3}$, Stefan Krivus ${ }^{1}$, Lubomir Straka ${ }^{2}$, Kristina Biskupska Bodova ${ }^{3}$ and Jan Danko ${ }^{3}$

\begin{abstract}
Background: Postpartum hemorrhage (PPH) represents a serious problem for women and obstetricians. Because of its association with hemorrhagic shock and predisposition to disseminated coagulopathy, it is a leading cause of maternal deaths worldwide. Furthermore, the jeopardy of PPH is rising with the secondary form of PPH occurring between 24 hours and 6 weeks postpartum, when women are already discharged home. The causes of this pathology are severe inflammation (endometritis), inherited coagulation disorders, consumptive coagulopathy, and retained products of conceptions. Others are of rare occurrence, such as vessel subinvolution (VSI) of the placental implantation site, uterine artery pseudoaneurysm, or trauma.

Case presentation: We present a rare form of recurrent secondary postpartum hemorrhage in a woman after uncomplicated cesarean delivery, with review of the literature linked to the management of this situation originating in the rare local VSI in the placental implantation site, defective decidual homeostasis, and coagulopathy confined to the uterus.

Conclusion: The placental site VSI is one of the rare causes of secondary PPH, and this situation is frequently underdiagnosed by clinicians. The histological confirmation of dilated "clustered"-shaped myometrial arteries partially occluded by thrombi of variable "age" together with the presence of endovascular extravillous trophoblasts confirms the diagnosis.
\end{abstract}

Keywords: Puerperium, Secondary postpartum hemorrhage, Vessel subinvolution, Coagulopathy

\section{Background}

Postpartum hemorrhage (PPH) represents serious problem for women and obstetricians. Because of its association with hemorrhagic shock and predisposition to disseminated coagulopathy, it is a leading cause of maternal deaths worldwide [1]. The severity of PPH is potentiated by the fact that it is not specifically associated with the mode of delivery (vaginal vs. cesarean section). Furthermore, the jeopardy of PPH is rising with the secondary form of $\mathrm{PPH}$ (abnormal excessive bleeding from the birth canal, mostly

\footnotetext{
* Correspondence: zubor@jfmed.uniba.sk

'Department of Obstetrics and Gynecology, University Hospital Martin, Kollarova 2, Martin 036 01, Slovakia

${ }^{3}$ Department of Obstetrics and Gynecology, Jessenius Faculty of Medicine,

Comenius University, Kollarova 2, Martin 036 01, Slovak Republic

Full list of author information is available at the end of the article
}

uterus, occurring between 24 hours and 6 weeks postpartum), when women are already discharged home.

$\mathrm{PPH}$ is a widely underestimated obstetric problem with variable occurrence and severity when diagnosed. This is caused by the lack of definitions and describing criteria used for this condition (e.g., quantification of blood loss, variable cutoff limits for estimated blood loss, and linkage to the mode of delivery). In general, the incidence of PPH is approximately $5 \%-20 \%$ of labors $[1,2]$, with the highest rates in developing countries. The WHO evidence is showing the wide geographic variability in $\mathrm{PPH}$; for example, in Africa, it accounts for 33.9\% of maternal deaths (interval 13.3-43.6\%), and in Asia, 30.8\% (intercountry interval: $5.9-48.5 \%)$. Apart from this major problem in the "third world" countries, the rising worries are observed recently also in developed countries like Australia,

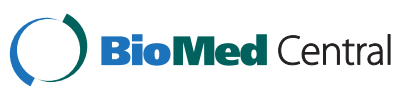


Belgium, Canada, France, Great Britain, or USA, which are showing increased incidence of $\mathrm{PPH}$ (from 1.9\% to $2.8 \%)[3,4]$. The causes of this trend are unknown, and we can only hypothesize the preconditions. However, causes can be the recent increase in the overall number of cesarean section, pertinently repeat cesarean section or IVF techniques with an increase in morbidly attached placenta pathologies. In general, it is the change in health-care management about pregnant women and labor conditions/circumstances. However, the common etiologies remain, such as placental abruption, transverse or classical cesarean delivery, manual placental extraction, uterine hypotony/atony, severe inflammation (endometritis), inherited coagulation disorders, consumptive coagulopathy, and retained products of conceptions [4]. Others are of sporadic occurrence and play an important role such as vessel subinvolution (VSI) of the placental implantation site $[5,6]$, trauma, or uterine artery pseudoaneurysm, where the affected vessel wall does not allow adequate contraction and involution. Under the physiological situation, there is spontaneous thrombi and fibrotic closure of the utero-placental vessels in the normal postpartum period. Involution process brings the vessels back to the nongestational state. Failure in this process may be associated with the transient reorganization of the vessel thrombi and their partial occlusion that can lead to severe profuse or intermittent vaginal bleeding after delivery [7], with rapid cardiovascular collapse necessitating sometimes urgent hysterectomy $[8,9]$. As mentioned previously, the wicked situation occurs when $\mathrm{PPH}$ is presented in its secondary form, even if it affects only $1-2 \%$ of postnatal women. This low incidence of secondary $\mathrm{PPH}$ and linkage to maternal morbidity rather than mortality was the reason for the little attention among obstetricians, but it is recently gaining importance and interest with the understanding of VSI pathology and maternal deaths because of this condition [10]. The primary danger for patient is that bleeding in the majority occurs between 1 and 2 weeks after delivery $[11,12]$ when patient is often home and unaware that the hemorrhage is significant and potentially life threatening.

In this paper, we present a rare form of recurrent secondary postpartum hemorrhage in a woman after uncomplicated cesarean delivery, with review of the literature linked to the management of this situation originating in the rare local VSI in placental implantation site, defective decidual homeostasis, and coagulopathy confined to the uterus.

\section{Case report}

A 24-year-old nulliparous primigravida woman was referred to our department in $40+3$ weeks' gestation for delivery with history of regular uterine contractions and fetal breech presentation (cervix dilated to $6 \mathrm{~cm}$, presenting part above the interspinal line). She underwent uncomplicated cesarean section (operative technique sec. Pfannenstiel-Geppert, uterus closed by double-layer interlocking separate resorbable sutures, followed by continuous suture of the visceral peritoneum) because of arrest of descent and signs of fetal intrauterine hypoxia. The estimated blood loss was $500 \mathrm{ml}$, and a prophylactic dose of the first-generation cephalosporin was administered intravenously. Antenatal course was uneventful, except untreated mild pregnancy anemia (hemoglobin $(\mathrm{Hb})$ : $102 \mathrm{~g} / \mathrm{L}$ ). Patient was discharged home on the 5th day postpartum with $\mathrm{Hb}$ level of $91 \mathrm{~g} / \mathrm{L}$, normal coagulation parameters, and a recommendation for iron supplementation and follow-up of blood count. On the 27th day postpartum, she experienced profuse vaginal bleeding with drop in the hemoglobin level (70 g/L), but physical and transvaginal uterine ultrasound examinations failed to reveal the cause (regular uterine involution, no signs of the retained placental tissue, or a visible anomaly of the cesarean scar). Conservative management with blood transfusion units (total volume $670 \mathrm{ml}$ ) and uterotonics (oxytocin, methylergometrine) was helpful to stabilize the patient, and she was discharged home within 4 days. Vaginal and cervical microbiological culture has showed disturbed vaginal flora with bacterial colonization of species: Escherichia coli, Corynebacterium species, Candida species, and Streptococcus agalactiae group B. However, recommended antibiotics therapy was not administered because of patient carelessness. On the 43rd day after delivery, she was readmitted with recurrent severe vaginal bleeding ( $\mathrm{Hb} 86 \mathrm{~g} / \mathrm{L})$. After initial blood transfusions (680 $\mathrm{ml}$ in total), the patient underwent hysteroscopy with curettage, showing no retained products of placental tissue or cesarean scar dehiscence. She was discharged home with blood count of $118 \mathrm{~g} / \mathrm{L}$, normal systemic coagulation parameters, and the recommendation of combined estrogen-progestin therapy (estrophem/2 mg/+ duphaston/dydrogesterone/10 mg daily) for early endometrium recovery. Despite this, on the 63rd day following delivery, the patient came back to the hospital with heavy life-threatening vaginal bleeding, signs of hemorrhagic shock (Hb level $61 \mathrm{~g} / \mathrm{L}$ ), and symptoms of hemodynamic instability (sweaty, pale looking, hypotension 90/50 $\mathrm{mmHg}$, and tachycardia 110 b.p.m.). She underwent emergency abdominal supravaginal hysterectomy. Macroscopic examination of the lower uterine segment showed no signs of hysterorrhaphy dehiscence during explorative laparotomy. Similarly, microscopic examination of the uterus and placental implantation site did not confirm the scare dehiscence but revealed large, dilated arteries containing partially occluding thrombi; trophoblastic cells within and surrounding the spiral arteries confirmed by immunohistochemistry; endometrial simplex hyperplasia; and infection (diffuse lymphomonocyte infiltrate) of the implantation 
site demonstrated by the presence of Escherichia coli, Corynebacterium species, Candida species, and Streptococcus agalactiae group B (Figures 1, 2, 3). Additionally, microscopic sections of the blood clot attached to the placental implantation site contained inflamed necrotizing decidua. No retained placenta or placenta accrete was noted on multiple sections. Moreover, the local intravascular coagulation disturbance confined to the uterus was suggested. The postoperative recovery was uneventful, and the patient was discharged after 11 days. One and four months later, she came for follow-up and was healthy.

\section{Discussion}

Postpartum hemorrhage is a serious obstetrics emergency occurring independently from the mode of delivery. The traditional definition of $\mathrm{PPH}$ is based on quantification of blood loss but has variable criteria and several limitations across countries or obstetrics national societies. In general, for mild PPH, a blood loss $>500 \mathrm{ml}$ and a severe blood loss $>1000 \mathrm{ml}$ are considered after spontaneous delivery [13] or above $1000 \mathrm{ml}$ in cesarean section [3]. Regardless of definitions used, only a visual assessment of blood loss is inaccurate [14], and many postpartum women are subsequently underdiagnosed/overdiagnosed for $\mathrm{PPH}$. Thus, alternative blood loss assessment is a check for a drop in hematocrit of $10 \%$ counted before and after delivery [15]. The bleeding after labor can occur within 24 hours (primary form of $\mathrm{PPH}$ ) or, later, from 24 hours after delivery until 6 weeks postpartum (secondary form) of PPH.

During the process of physiological subinvolution, bleeding from the uterus is regulated by several factors and mechanisms, for example, myometrial contraction and local decidual and systemic coagulation factors leading to the minimalization of blood loss after delivery. Moreover, at the end of the third trimester, the extravillous

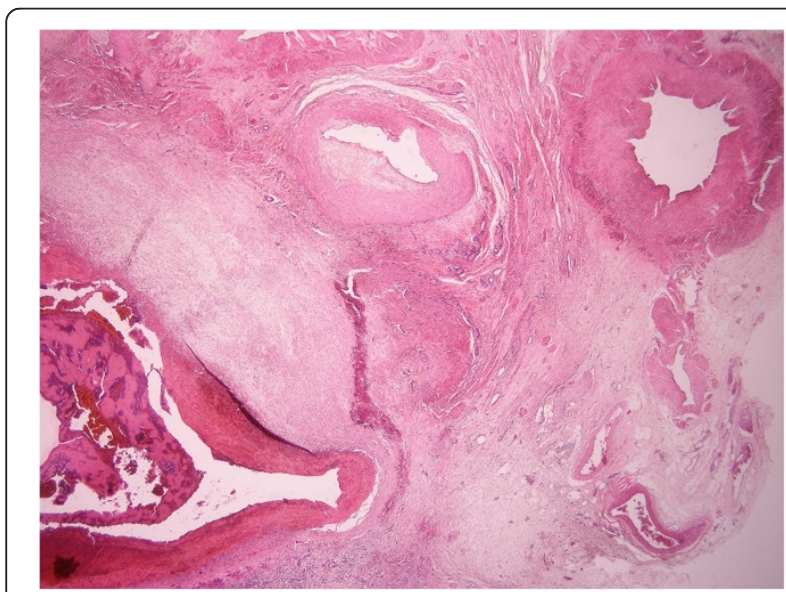

Figure 1 Local placental site vessel subinvolution (right above) with active bleeding (left down). (H\&E staining, magnification: $\times 100$ )

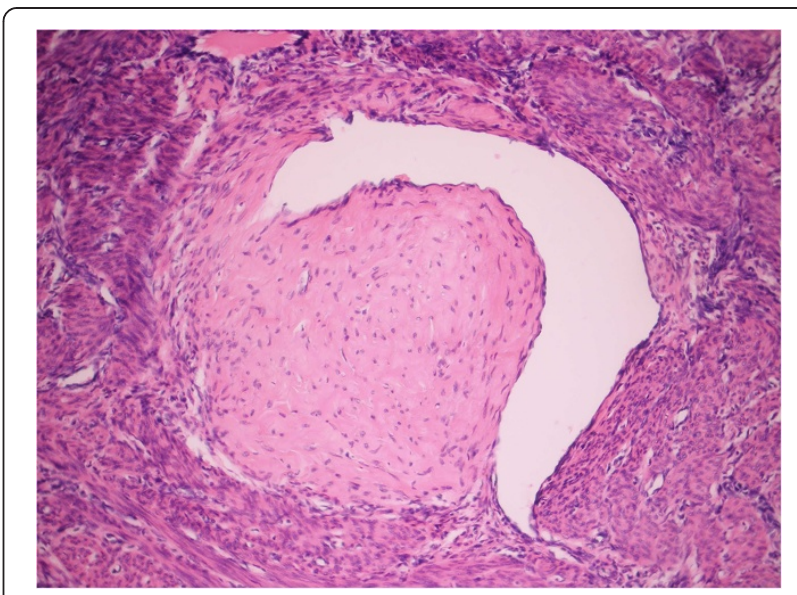

Figure 2 Detailed view on subinvoluted vessel with partial obliteration by thrombi (H\&E staining, magnification: $\times 400$ ).

endovascular trophoblast (EVCT) for maternal endothelial cells in myometrial vessels located in the placental site is starting to be replaced. With this vascular involution, change is playing a role also in the occlusive form of the intima layer thickening via its fibrotic transformation, local endarteritis, thrombotization, and regeneration of the internal elastic lamina of the uterus vascular system.

In pathology, for example, with abnormal complement component activity or with the presence of long-lasting expression of apoptotic genes as $\mathrm{Bcl}-2$ inhibiting apoptosis and supporting cell survival, [16] VSI develops (luminal partial occlusion of the uterine vessels) in the previous placental site implantation. This would result in temporary occlusion of the vessels (mainly spiral arteries in the superficial myometrium at the placental implantation site) where the newly formed thrombi undergo resorption within a period of 2-3 weeks, which can lead to recurrent

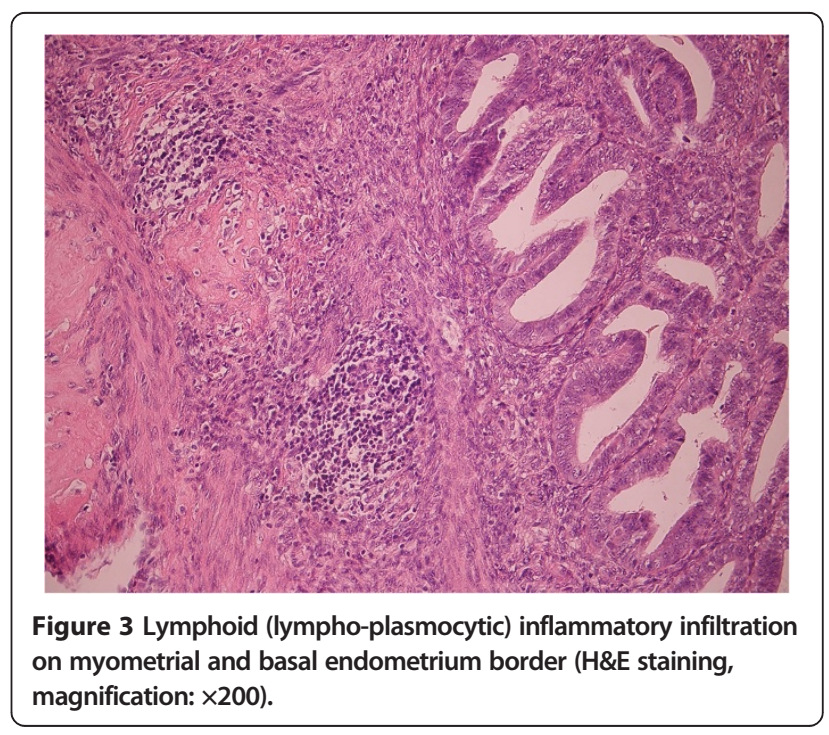


uterine bleeding and enlarged and boggy uterus. The possibility of such phenomenon is becoming more obvious when bleeding reoccurs in regular periods. In our case, the uterine bleeding recurred in a period of 15-20 days (27th, 43rd, and 63rd day postpartum). The severity of bleeding can be augmented by the presence of active inflammation (confirmed also in our case), which may enhance through the production of proinflammatory factors of the local tissue coagulopathy [17] and switch to the chronicity with the development of chronic disseminated intravascular coagulation $[18,19]$. Such "terrain" is predisposed to secondary PPH. The situation observed also by our study.

To diagnose the placental site VSI with the presence of local site inflammation and tissue coagulopathy is difficult. The exact directed histological examination of bioptic material from the previous placental site can be helpful. The presence of vascular subinvolution can be confirmed by the finding of EVCT. Unfortunately, we did not observe this in our histological specimens that were obtained from hysteroscopy and curettage. In this situation, we have many other clinicians working under pressure and focused more on finding potential myometrium scar dehiscence and observing the condition of the endometrium (hyperplasia after hormonal replacement therapy). Omitting detection of EVCT and other possible helpful markers as immunostaining positivity for cytokeratin profile, inhibin alpha, Mel-CAM, Bcl-2, elastin, complement components, or human placental lactogen is a mistake as they are enriching the information for the direct diagnosis of VSI. Moreover, the histological evidence of VSI is also the presence of complement cascade markers $(\mathrm{C} 1 \mathrm{q}$, C3d, C4, and C9) or immunoglobulins G, A, and M [20]. However, although all of these markers can be evaluated, they did not explain the basic molecular mechanism of placental site subinvolution. Surgical pathologist must therefore be aware of the major histopathologic findings of VSI in every postpartum curettage or hysterectomy specimens.

The exact pathophysiology of VSI is not known. Some suspect an immune component leading to abnormal interaction between the maternal uterine cells and fetal trophoblast [5]; others, as mentioned above, altered complement components activity or the presence of longlasting expression of apoptotic genes (e.g., Bcl-2), inhibiting apoptosis [16]. However, after the review of the literature, there is one specific situation that is related to secondary postpartum hemorrhage and published in MEDLINE. The search in this register (June 2011) under keywords "secondary or delay or unusual cause of postpartum hemorrhage" showed 18 papers dealing with secondary postpartum hemorrhage where 9 papers [7,10,21-27] reported cases of secondary PPH after cesarean section with two major conclusions: placental site VSI or uterine artery pseudoaneurysm. To explain how cesarean section enhances the higher occurrence of VSI is difficult. One may only speculate that some mechanic (e.g., altered myometrium contraction and involution, fibrotic-proliferation in scar), immune (autoimmune and healing processes, reaction to suturing material used, local viral or bacterial inflammation, role of lymphomonocytic and cytokine activity), or molecular mechanisms like overexpression and underexpression of growth factor genes involved in the uterine scarring process, genes modifying the decidua replacement by regular endometrium, collagen deposition in the vessels of wound site, platelet aggregation, factor XIII activity, and gene expressions of pro-angiogenic and anti-angiogenic factors and receptors at the maternal-fetal interface are playing a role with a consequent secondary postpartum hemorrhage [28-31].

Although the incidence of secondary postpartum hemorrhage after cesarean section is very low (app. 0.1\%) [22], therapeutic management is close to primary $\mathrm{PPH}$ and requires coordination and multidisciplinary care, aiming the immediate hemodynamic stabilization of the patient, depleted blood volume, and development of coagulopathy. Treatment usually falls into one of two options: surgical evacuation of the uterine cavity or medical treatment [32]. Often, blood and plasma unit transfusion is required. Speculum examination of the cervix and the lower genital tract to exclude possible lacerations is obligatory. Furthermore, uterine ultrasound is mandatory to exclude a possibility of the retained placental tissue [33]. In differential diagnosis, it is necessary to exclude vaginal bleeding because of the severe endometritis, retained placental tissue, or gestational trophoblastic disease, where laboratory findings of inflammatory markers (e.g., CRP, leucocytes), positive blood and vaginal cultures, elevated $\beta$ HCG levels, and expert ultrasound examination [33,34] are essential for the adequate diagnosis.

\section{Conclusions}

In conclusion, we can say that placental site VSI is one of the rare forms of secondary PPH and is frequently underdiagnosed by clinicians. The histological confirmation of dilated and "clustered"-shaped myometrial arteries partially occluded by thrombi of variable "age," together with the presence of endovascular extravillous trophoblast, confirms the diagnosis. However, this is done mainly after the urgent hysterectomy. Furthermore, we believe that this type of secondary PPH is of idiopathic than of iatrogenic cause, and our previously published [35] data prove that there are no known predictive factors for this pathology (e.g., clinical assessment with/without transabdominal ultrasound). The obstetricians and caregivers are demanding for an early recognition of severe forms of a secondary $\mathrm{PPH}$, thus emphasizing that VSI in the placental implantation site could be beneficial for targeted therapy and may preserve woman's fertility. 


\section{Consent}

Written informed consent was obtained from the patient for publication of this Case report and any accompanying images. A copy of the written consent is available for review by the Editor of this journal.

\section{Competing interests}

The authors report no declarations of interest.

\section{Authors' contributions}

$\mathrm{PZ}$ and KD contributed equally to write up of this case report. SK and KBB were involved in the clinical management of this patient. KK and LS were involved in histopathological analysis. JD interpreted the data and critically revised the manuscript. All authors have read and approved the final manuscript.

\section{Acknowledgement}

This work was supported by the ITMS no. 26220120036 project "Centre of excellence for perinatology research II" supported by EU sources. We would like to thank Jozef Kornan, M.D. for the personal assistance in patient management and data collection.

\section{Author details}

'Department of Obstetrics and Gynecology, University Hospital Martin, Kollarova 2, Martin 036 01, Slovakia. ²Department of Pathology, University Hospital Martin, Kollarova 2, Martin 036 01, Slovakia. ${ }^{3}$ Department of Obstetrics and Gynecology, Jessenius Faculty of Medicine, Comenius University, Kollarova 2, Martin 036 01, Slovak Republic.

Received: 26 February 2013 Accepted: 16 January 2014

Published: 21 February 2014

\section{References}

1. Carroli G, Cuesta C, Abalos E, Gulmezoglu AM: Epidemiology of postpartum haemorrhage: a systematic review. Best Pract Res Clin Obstet Gynaecol 2008, 22:999-1012.

2. Khan KS, Wojdyla D, Say L, Gülmezoglu AM, Van Look PF: WHO analysis of causes of maternal death: a systematic review. Lancet 2006, 367:1066-1074.

3. Knight M, Callaghan WM, Berg C, Alexander S, Bouvier-Colle MH, Ford JB, Joseph KS, Lewis G, Liston RM, Roberts CL, Oats J, Walker J: Trends in postpartum hemorrhage in high resource countries: a review and recommendations from the International Postpartum Hemorrhage Collaborative Group. BMC Pregnancy Childbirth 2009, 9:55.

4. Fong A, Leake J, Pan D, Ogunyemi D: Demographic, institutional and obstetrical risk factors for postpartum haemorrhage mortality. J Obstet Gynaecol 2010, 30:470-475.

5. Andrew AC, Bulmer JN, Wells M, Morrison L, Buckley $\mathrm{CH}$ : Subinvolution of the uteroplacental arteries in the human placental bed. Histopathology 1989, 15:395-405.

6. Weydert JA, Benda JA: Subinvolution of the placental site as an anatomic cause of postpartum uterine bleeding: a review. Arch Pathol Lab Med 1994, 130:1538-1542.

7. Al-Mehaisen L, Al-Kuran O, Amarin ZO, Matalka I, Beitawi S, Muhtaseb A: Secondary postpartum hemorrhage following placental site vessel subinvolution: a case report. Arch Gynecol Obstet 2008, 278:585-587.

8. Christopoulos P, Hassiakos D, Tsitoura A, Panoulis K, Papadias K, Vitoratos N: Obstetric hysterectomy: review of cases over 16 years. J Obstet Gynaecol 2011, 31:139-141.

9. Tadesse W, Farah N, Hogan J, D'Arcy T, Kennelly M, Turner MJ: Peripartum hysterectomy in the first decade of the 21st century. J Obstet Gynaecol 2011, 31:320-321.

10. Farley NJ, Kohlmeier RE: A death due to subinvolution of the uteroplacental arteries. J Forensic Sci 2011, 56:803-805.

11. King PA, Duthie SJ, Dong ZG, Ma HK: Secondary postpartum haemorrhage. Aust N Z J Obstet Gynaecol 1989, 29:394-398.

12. Hoveyda F, MacKenzie IZ: Secondary postpartum haemorrhage: incidence, morbidity and current management. BJOG 2001, 108:927-930.

13. Bais JM, Eskes M, Pel M, Bonsel GJ, Bleker OP: Postpartum haemorrhage in nulliparous women: incidence and risk factors in low and high risk women. A Dutch population-based cohort study on standard (> or $=500 \mathrm{ml}$ ) and severe $(>$ or $=1000 \mathrm{ml}$ ) postpartum haemorrhage. Eur J Obstet Gynecol Reprod Biol 2004, 115:166-172.

14. Stafford I, Dildy GA, Clark SL, Belfort MA: Visually estimated and calculated blood loss in vaginal and cesarean delivery. Am J Obstet Gynecol 2008, 199:519. e1-7.

15. Combs CA, Murphy EL, Laros RK Jr: Factors associated with postpartum hemorrhage with vaginal birth. Obstet Gynecol 1991, 77:69-76.

16. Khong TY, Abdul Rahman $\mathrm{H}$ : Bcl-2 expression delays postpartum involution of pregnancy-induced vascular changes in the human placental bed. Int J Gynecol Pathol 1997, 16:138-142.

17. Zrubek H, Sikorski M: Local coagulation confined to the uterus and adnexa-case description. Ginekol Pol 1999, 4(65):593-595.

18. Somashekhar M, Kadamba PS, Wakodkar M: Chronic disseminated intravascular coagulation presenting as renal mass. J Indian Assoc Pediatr Surg 2008, 13:144-146.

19. Cai B, Deitch EA, Ulloa L: Novel insights for systemic inflammation in sepsis and hemorrhage. Mediators Inflamm 2010, 2010:642462.

20. Andrew A, Bulmer JN, Morrison L, Wells M, Buckley CH: Subinvolution of the uteroplacental arteries: an immunohistochemical study. Int J Gynecol Pathol 1993, 12:28-33.

21. Keane B: Recurrent secondary postpartum haemorrhage after lower segment caesarean section. N Z Med J 1973, 79:256-257.

22. Shweni PM, Bishop BB, Hansen JN, Subrayen KT: Severe secondary postpartum haemorrhage after caesarean section. S Afr Med J 1987, 72:617-619.

23. Paraskevaides E, Stuart B, Gardeil F: Secondary postpartum haemorrhage from nondehisced lower caesarean section scar: a case for hysteroscopy. Aust N Z J Obstet Gynaecol 1993, 33:427.

24. Pollio F, Staibano S, De Falco M, Buonocore U, De Rosa G, Di Lieto A: Severe secondary postpartum hemorrhage 3 weeks after cesarean section: alternative etiologies of uterine scar non-union. J Obstet Gynaecol Res 2007, 33:360-362.

25. Lausman AY, Ellis CA, Beecroft JR, Simons M, Shapiro JL: A rare etiology of delayed postpartum hemorrhage. J Obstet Gynaecol Can 2008, 30:239-243.

26. Samad S, Jonetzko P, Hawkins AP, Booth J, Thorpe-Beeston G, Marwood R: An unusual cause of delayed postpartum haemorrhage following caesarean section. Emerg Med J 2009, 26:621-622.

27. Majumdar A, Bird A: A case of recurrent secondary postpartum haemorrhage treated by uterine artery embolisation. J Obstet Gynaecol 2010, 30:412-413.

28. Boscolo E, Bischoff J: Vasculogenesis in infantile hemangioma. Angiogenesis 2009, 12:197-207.

29. Petitbarat M, Serazin V, Dubanchet S, Wayner R, de Mazancourt P, Chaouat G, Lédée N: Tumor necrosis factor-like weak inducer of apoptosis (TWEAK)/ fibroblast growth factor inducible-14 might regulate the effects of interleukin 18 and 15 in the human endometrium. Fertil Steril 2010, 94:1141-1133.

30. Edwards AK, van den Heuvel MJ, Wessels JM, Lamarre J, Croy BA, Tayade C: Expression of angiogenic basic fibroblast growth factor, platelet derived growth factor, thrombospondin-1 and their receptors at the porcine maternal-fetal interface. Reprod Biol Endocrinol 2011, 9:5

31. Gosk-Bierska I, McBane RD, Wu Y, Mruk J, Tafur A, McLeod T, Wysokinski WE: Platelet factor XIII gene expression and embolic propensity in atrial fibrillation. Thromb Haemost 2011, 106:75-82.

32. Neill A, Thornton S: Secondary postpartum haemorrhage. J Obstet Gynaecol 2002, 22:119-122.

33. Zubor P, Szunyogh N, Dokus K, Scasny P, Kajo K, Galo S, Biringer K, Krivus S, Danko J: Application of uterotonics on the basis of regular ultrasonic evaluation of the uterus prevents unnecessary surgical intervention in the postpartum period. Arch Gynecol Obstet 2010, 282:261-267.

34. Mulic-Lutvica A, Eurenius K, Axelsson O: Uterine artery Doppler ultrasound in postpartum women with retained placental tissue. Acta Obstet Gynecol Scand 2009, 88:724-728.

35. Neill AC, Nixon RM, Thornton S: A comparison of clinical assessment with ultrasound in the management of secondary postpartum haemorrhage. Eur J Obstet Gynecol Reprod Biol 2002, 104:113-115.

doi:10.1186/1471-2393-14-80

Cite this article as: Zubor et al:: Recurrent secondary postpartum hemorrhages due to placental site vessel subinvolution and local uterine tissue coagulopathy. BMC Pregnancy and Childbirth 2014 14:80. 